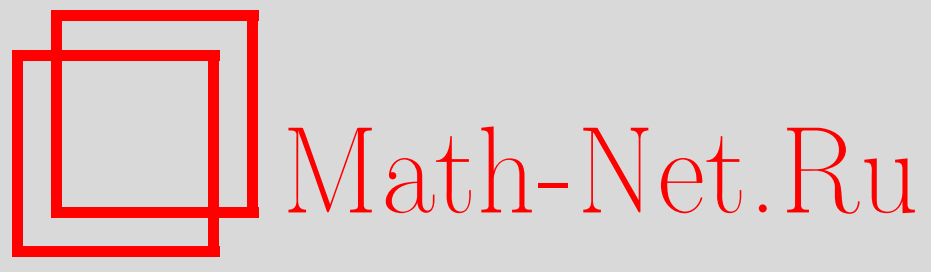

М. В. Зайцев, С. П. Мищенко, Рост некоторых многообразий супералгебр Ли, Изв. РАН. Сер. матем., 2007, том 71, выпуск 4, 3-18

DOI: https://doi.org/10.4213/im689

Использование Общероссийского математического портала Math-Net.Ru подразумевает, что вы прочитали и согласны с пользовательским соглашением http://www . mathnet.ru/rus/agreement

Параметры загрузки:

IP : 54.92.164.108

26 апреля 2023 г., 11:50:18

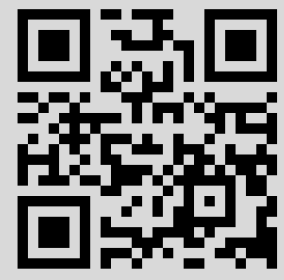


УДК 512.8

\author{
М. В. Зайцев, С. П. Мищенко
}

\title{
Рост некоторых многообразий супералгебр Ли
}

Изучаются числовые характеристики многообразий супералгебр Ли, в частности рост коразмерностей. Построен пример неразрешимого многообразия почти полиномиального роста. Доказано, что экспонента построенного многообразия равна трем. Кроме того, вычислены экспоненты роста двух известных ранее разрешимых многообразий.

Библиография: 16 наименований.

\section{§ 1. Введение}

В статье изучается рост многообразий супералгебр Ли над полем нулевой характеристики (см. [1]).

Напомним необходимые определения. Пусть $F$ - свободная неассоциативная алгебра, порожденная счетным множеством $X=\left\{x_{1}, x_{2}, \ldots\right\}$ над полем $\Phi$, $\operatorname{char} \Phi=0$, a V - некоторое многообразие $\Phi$-алгебр. Тогда совокупность тождеств многообразия $\mathbf{V}$ образует идеал $I$ в $F$. Обозначим через $P_{n}$ подпространство полилинейных элементов от $x_{1}, \ldots, x_{n}$ в $F$. Положим

$$
\gamma_{n}(\mathbf{V})=\operatorname{dim} \frac{P_{n}}{P_{n} \cap I}
$$

Последовательность $\left\{\gamma_{n}(\mathbf{V})\right\}, n=1,2, \ldots$, является числовой характеристикой многообразия $\mathbf{V}$. Понятно, что можно рассматривать пространство $P_{n}(\mathbf{V})$ полилинейных элементов степени $n$ относительно свободной алгебры $F=F(X, \mathbf{V})$ многообразия $\mathbf{V}$. Действие $\sigma\left(x_{i}\right)=x_{\sigma(i)}$ симметрической группы $S_{n}$ естественным образом продолжается до автоморфизма алгебры $F=F(X, \mathbf{V})$. Пространство $P_{n}(\mathbf{V})$ становится при этом $S_{n}$-модулем. Исследование структуры $P_{n}(\mathbf{V})$ как $S_{n}$-модуля играет важную роль при изучении многообразия $\mathbf{V}$. Этот модуль является вполне приводимым. Рассмотрим разложение его характера в целочисленную комбинацию неприводимых характеров:

$$
\chi_{n}(\mathbf{V})=\chi\left(P_{n}(\mathbf{V})\right)=\sum_{\lambda \vdash n} m_{\lambda} \chi_{\lambda} .
$$

В случае алгебр Ли или ассоциативных алгебр размерность пространства полилинейных элементов относительно свободной алгебры, как принято, будем обозначать $c_{n}(\mathbf{V})$ вместо $\gamma_{n}(\mathbf{V})$. В случае если многообразие $\mathbf{V}$ порождается алгеброй $A$, т. е. $\mathbf{V}=\operatorname{var} A$, то будем использовать обозначение $\gamma_{n}(A)$ или $c_{n}(A)$.

ЗАмЕчаниЕ 1. Если V - многообразие Ф-супералгебр Ли, то можно рассмотреть еще одну числовую характеристику. Обозначим через $\mathcal{L}=\mathcal{L}_{0} \oplus \mathcal{L}_{1}$ свободную супералгебру Ли над $\Phi$ с тем же множеством $X$ четных и множеством $Y=\left\{y_{1}, y_{2}, \ldots\right\}$ нечетных порождающих. Обозначим через $R$ неоднородную

Работа частично поддержана РФФИ (гранты № 07-01-00080 и № 06-01-00485) и программой "Ведущие научные школы” (грант НШ-5666.2006.1).

(C) М. В. ЗАйцев, С. П. Мищенко, 2007 
подалгебру в $\mathcal{L}$, порожденную элементами $z_{i}=x_{i}+y_{i}, i=1,2, \ldots$, а через $\bar{P}_{n}-$ подпространство полилинейных полиномов от $z_{1}, \ldots, z_{n}$ в $R$. Любое отображение $x_{i} \mapsto a_{i} \in L_{0}, y_{i} \mapsto b_{i} \in L_{1}$ со значениями в некоторой супералгебре Ли $L=L_{0} \oplus L_{1}$ однозначно продолжается до гомоморфизма $R \rightarrow L$. Если $L-$ свободная алгебра счетного ранга многообразия $\mathbf{V}$, то пересечение $J$ ядер всех гомоморфизмов из $R$ в $L$ можно также интерпретировать как идеал тождеств многообразия $\mathbf{V}$ и рассматривать вместо $\gamma_{n}(\mathbf{V})$ коразмерности подпространств $\bar{P}_{n} \cap J$ в $\bar{P}_{n}$. Однако эти величины совпадают, поскольку для канонического эпиморфизма $\pi: F \rightarrow R$, где $\pi\left(x_{i}\right)=z_{i}$, выполняются равенства $\pi\left(P_{n}\right)=\bar{P}_{n}$, $\pi(I)=J, \pi^{-1}(J)=I$. Это позволяет проводить вычисления как в свободной неассоциативной алгебре, так и в свободной супералгебре Ли.

Рост многообразия $\mathbf{V}$ называют полиномиальным, если $\gamma_{n}(\mathbf{V})<n^{k}$ при достаточно больших $n$, и экспоненциальным, если $a^{n}<\gamma_{n}(\mathbf{V})<b^{n}$ для некоторых $a, b>1$ при больших $n$. Если $\gamma_{n}(\mathbf{V})$ асимптотически больше $a^{n}$ для любого заданного $a$, то $\mathbf{V}$ принято называть многообразием сверхэкспоненииального роста. Например, если $\mathbf{V}$ - многообразие всех ассоциативных $\Phi$-алгебр, то $\gamma_{n}(\mathbf{V})=n$ !. Если $\mathbf{V}$ - многообразие всех алгебр Ли, то $\gamma_{n}(\mathbf{V})=(n-1)$ !. В случае ассоциативных алгебр хорошо известен результат А. Регева [2], согласно которому $\gamma_{n}(\mathbf{V})<a^{n}$ для некоторого $a>1$, если $\mathbf{V}$ - нетривиальное многообразие ассоциативных алгебр. В классе лиевских многообразий реализуются и сверхэкспоненциальные функции роста (см., например, [3], [4]). Однако общим для ассоциативных, лиевских, а также для супералгебр Ли оказалось отсутствие многообразий промежуточного роста таких, что функция $\gamma_{n}(\mathbf{V})$ растет быстрее любого полинома, но медленнее любой экспоненты (см., например, [1], [5], [6]). Отметим также, что к настоящему времени многообразия полиномиального роста описаны в терминах тождественных соотношений как в ассоциативном случае [7], так и в лиевском [8], [9], и в суперлиевском случае [1].

Определим еще одну важную числовую характеристику многообразия линейных алгебр: так называемую экспоненту многообразия. В случае, когда последовательность коразмерностей $\gamma_{n}(\mathbf{V}), n=1,2, \ldots$, экспоненциально ограничена, т. е. $\gamma_{n}(\mathbf{V}) \leqslant d^{n}$, рассмотрим ограниченную последовательность $\sqrt[n]{\gamma_{n}(\mathbf{V})}, n=1,2, \ldots$ В случае существования предела $\lim _{n \rightarrow \infty} \sqrt[n]{\gamma_{n}(\mathbf{V})}$ назовем его экспонентой многообразия, которую обозначим $\operatorname{Exp}(\mathbf{V})$.

В упомянутой работе авторов [1] были представлены два примера многообразий почти полиномиального роста: многообразие метабелевых супералгебр Ли и многообразие, порожденное алгеброй $H$, где $H=H_{0} \oplus H_{1}$ - четырехмерная супералгебра Ли с базисом $a, b, u, v$ и таблицей умножения $a b=0, u v=0$, $a u=v, a v=u, b u=u, b v=v, a^{2}=2 b, b^{2}=u^{2}=v^{2}=0$, в которой $H_{0}=\langle b, v\rangle$, $H_{1}=\langle a, u\rangle$.

Основным результатом настоящей работы является построение нового примера многообразия почти полиномиального роста. Этим примером является многообразие, порожденное грассмановой оболочкой $\mathbb{Z}_{2}$-градуированной трехмерной алгебры Ли $\mathrm{sl}^{\mathrm{gr}}(2, \Phi)$ матриц порядка два с нулевым следом. Кроме того, будут найдены экспоненты двух известных упомянутых выше примеров многообразий почти полиномиального роста.

\section{§ 2. Вспомогательные результаты}

Договоримся не использовать скобки в случае их левонормированной расстановки, т. е. $u_{1} u_{2} u_{3}=\left(u_{1} u_{2}\right) u_{3}$. Будем использовать обозначение $a b^{n}$ для левонормированного произведения $a b \cdots b$, в котором $b$ встречается $n$ раз. На- 
помним общепринятые обозначения некоторых многообразий супералгебр Ли. Многообразие, определяемое тождеством $x_{1} x_{2} \equiv 0$, обозначим $\mathbf{A}$. Тождество $x_{1} x_{2} \cdots x_{c+1} \equiv 0$ определяет многообразие $\mathbf{N}_{c}$. Многообразие $\mathbf{A}^{2}$ определяется тождеством $\left(x_{1} x_{2}\right)\left(x_{3} x_{4}\right) \equiv 0$, a $\mathbf{N}_{c} \mathbf{A}-$ тождеством $\left(x_{1} x_{2}\right)\left(x_{3} x_{4}\right) \times \cdots$ $\cdots \times\left(x_{2 c+1} x_{2(c+1)}\right) \equiv 0$.

Как упоминалось во введении, в статье [1] был найден критерий полиномиальности роста многообразий супералгебр Ли. Этот критерий будет регулярно использоваться в настоящей работе, поэтому для удобства читателей приводим формулировку этого результата.

Теорема 1 [1]. Многообразие V супералгебр Ли над полем нулевой характеристики имеет полиномиальный рост тогда и толъко тогда, когда выполняются следующие условия:

1) $\mathbf{V} \subset \mathbf{N}_{c} \mathbf{A}$

2) существует такое $k$, что любой полилинейный полином, содержащий не менее $k$ четных и $k$ нечетных переменных, тождественно равен нулю в $\mathbf{V}$;

3) в многообразии $\mathbf{V}$ для любого $r=0,1, \ldots, c-1$ выполняются тождества вида

$$
\begin{aligned}
& \left(z_{1} z_{2} x_{1} \cdots x_{N}\right) t_{1} \cdots t_{r}\left(z_{3} z_{4} x_{N+1} \cdots x_{2 N}\right) \\
& \quad \equiv \sum_{\sigma} \alpha_{\sigma}^{r}\left(z_{1} z_{2} x_{\sigma(1)} \cdots x_{\sigma(N)}\right) t_{1} \cdots t_{r}\left(z_{3} z_{4} x_{\sigma(N+1)} \cdots x_{\sigma(2 N)}\right)
\end{aligned}
$$

где сумма берется лишь по тем подстановкам $\sigma \in S_{2 N}$, для которых $\sigma(\{1, \ldots$ $\ldots, N\}) \nsubseteq\{1, \ldots, N\}$.

Пусть $L=L_{0}+L_{1}$ - относительно свободная $\mathbb{Z}_{2}$-градуированная алгебра Ли некоторого многообразия градуированных алгебр Ли $\mathbf{V}$ с порождающими $x_{1}, x_{2}, \ldots \in L_{0}, y_{1}, y_{2}, \ldots \in L_{1}$. Обозначим пространство полилинейных элементов от $x_{1}, \ldots, x_{k}, y_{k+1}, \ldots, y_{n}$ этой алгебры через $P_{k, n-k}(\mathbf{V})$, а в случае свободной градуированной алгебры Ли просто $P_{k, n-k}$.

Определим отображение $\Theta$ из полилинейной компоненты $P_{k, m}$ свободной градуированной алгебры Ли $L(X, Y)$ с множеством четных свободных образующих $X=\left\{x_{1}, x_{2}, \ldots\right\}$ и нечетных $Y=\left\{y_{1}, y_{2}, \ldots\right\}$ в свободную супералгебру Ли $\mathcal{L}=\mathcal{L}_{0} \oplus \mathcal{L}_{1}$ с тем же множеством $X$ четных и тем же множеством $Y$ нечетных порождающих.

Для одночлена из свободной градуированной алгебры

$$
f\left(x_{1}, \ldots, x_{k}, y_{1}, \ldots, y_{m}\right)=\cdots y_{p(1)} \cdots y_{p(2)} \cdots y_{p(m)} \cdots,
$$

где $p$ - некоторая перестановка, результат действия будет иметь вид

$$
(\Theta f)\left(x_{1}, \ldots, x_{k}, y_{1}, \ldots, y_{m}\right)=(-1)^{p} \cdots y_{p(1)} \cdots y_{p(2)} \cdots y_{p(m)} \cdots
$$

Распространим действие по линейности на любой элемент полилинейной части. Непосредственной проверкой можно убедиться в справедливости следующего утверждения.

ПрЕДЛОЖЕНИЕ 1. Отображение $\Theta$ задает биекиию между многообразиями $\mathbb{Z}_{2}$-градуированных алгебр Ли и многообразиями супералгебр Ли. Кроме того, если $V_{1} \subset V_{2}-$ два различных многообразия $\mathbb{Z}_{2}$-градуированных алгебр Ли, то $\Theta\left(V_{1}\right) \subset \Theta\left(V_{2}\right)$ - различные многообразия супералгебр Ли. 
Заметим также, что отображение $\Theta$ можно определить и в обратную сторону: из полилинейной компоненты свободной супералгебры Ли в свободную алгебру Ли.

Обозначим через $G$ алгебру Грассмана со счетным числом образующих $g_{i}$, $i \in \mathbb{N}$. Алгебра $G$ имеет естественную $\mathbb{Z}_{2}$-градуировку $G=G_{0}+G_{1}$, где $G_{0}-$ пространство элементов четной степени от образующих, а $G_{1}$ - нечетной степени. Напомним, что для $\mathbb{Z}_{2}$-градуированной алгебры Ли $L=L_{0}+L_{1}$ алгебра $G(L)=L_{0} \otimes G_{0}+L_{1} \otimes G_{1} \subset L \otimes G$ является супералгеброй Ли и называется грассмановой оболочкой алгебры $L$.

ПредЛОЖЕНИЕ 2. Пусть $L=L_{0}+L_{1}-\mathbb{Z}_{2}$-градуированная алгебра Ли и $U=G(L)$ - ее грассманова оболочка. Тогда $\gamma_{n}(U) \leqslant 2^{n} c_{n}(L)$. Если дополнительно существует такое $k$, что $f\left(x_{1}, \ldots, x_{m}, y_{1}, \ldots, y_{t}\right)=0$ в L для любъх $x_{1}, \ldots, x_{m} \in L_{0}, y_{1}, \ldots, y_{t} \in L_{1}$ при условии, что $m, t>k$, то $\gamma_{n}(U) \leqslant \alpha n^{k} c_{n}(L)$ для некоторой константы $\alpha$.

ДокАЗАтельство. Заметим сначала, что если $A, B$ - две $\mathbb{Z}_{2}$-градуированные алгебры Ли с одинаковыми градуированными тождествами, то и супералгебры Ли $G(A)$ и $G(B)$ имеют одни и те же тождества. Поэтому алгебру $L$ можно считать относительно свободной с порождающими $x_{1}, x_{2}, \ldots \in L_{0}$, $y_{1}, y_{2}, \ldots \in L_{1}$. Пусть алгебра Грассмана $G$ порождена элементами $g_{1}, g_{2}, \ldots$ Рассмотрим в $U=G(L)$ подалгебру, порожденную $v_{1}, v_{2}, \ldots$, где $v_{i}=x_{i} \otimes$ $g_{3 i-2} g_{3 i-1}+y_{i} \otimes g_{3 i}, i=1,2, \ldots$ Хотя эта алгебра и не является относительно свободной в $\operatorname{var} U$, любое равное нулю полилинейное соотношение на $v_{1}, v_{2}, \ldots, v_{n}$ является тождеством в $G(L)$. Поэтому $\gamma_{n}(U)$ равно числу линейно независимых полилинейных полиномов от $v_{1}, v_{2}, \ldots, v_{n}$.

Обозначим через $W_{n}$ векторное пространство полилинейных одночленов от $z_{1}, z_{2}, \ldots, z_{n}$, где $z_{i}=x_{i}+y_{i}$, в алгебре Ли $L$, а для любого набора $t=\left(t_{1}, \ldots, t_{n}\right)$ длиной $n$ из нулей и единиц через $W_{n}^{t}$ обозначим линейную оболочку левонормированных произведений вида

$$
w_{\sigma(1)} \cdots w_{\sigma(n)}, \quad \sigma \in S_{n},
$$

в которой $w_{i}=x_{i}$, если $t_{i}=0$, и $w_{i}=y_{i}$, если $t_{i}=1$. Тогда

$$
W_{n} \subseteq \sum_{t \in \mathbb{Z}_{2}^{n}} W_{n}^{t}
$$

Заметим, что размерность любого подпространства $W_{n}^{t}$ не превосходит $c_{n}(L)$. Отсюда следует первое утверждение предложения.

Пусть теперь любое произведение в алгебре $L$, содержащее не менее $k$ четных и не менее $k$ нечетных переменных, равно нулю. Тогда количество ненулевых слагаемых $W_{n}^{t}$ в правой части $(2)$ не превосходит $2 \cdot\left(\begin{array}{l}n \\ k\end{array}\right)=\frac{2 n !}{k !(n-k) !}$, откуда следует второе утверждение предложения.

\section{§ 3. Неразрешимые многообразия почти полиномиального роста}

Рассмотрим алгебру Ли sl $(2, \Phi)$ квадратных матриц порядка два с нулевым следом. Пусть

$$
e=\left(\begin{array}{ll}
0 & 1 \\
0 & 0
\end{array}\right), \quad f=\left(\begin{array}{ll}
0 & 0 \\
1 & 0
\end{array}\right), \quad h=\left(\begin{array}{cc}
1 & 0 \\
0 & -1
\end{array}\right)
$$


- стандартный базис этой алгебры. Положим

$$
(\operatorname{sl}(2, \Phi))_{0}=\operatorname{Span}\langle h\rangle, \quad(\operatorname{sl}(2, \Phi))_{1}=\operatorname{Span}\langle e, f\rangle .
$$

Тогда $\mathrm{sl}^{\mathrm{gr}}(2, \Phi)=(\operatorname{sl}(2, \Phi))_{0}+(\operatorname{sl}(2, \Phi))_{1}$ является $\mathbb{Z}_{2}$-градуированной алгеброй Ли.

Для исследования многообразия супералгебр Ли, порожденного грассмановой оболочкой построенной градуированной алгебры, нам потребуются результаты о строении многообразия градуированных алгебр Ли, порожденного самой алгеброй $\mathbf{V}_{0}=\operatorname{var}\left(\operatorname{sl}^{\mathrm{gr}}(2, \Phi)\right)$.

Если $L$ - относительно свободная алгебра многообразия, то обозначим через $\left(L^{2}\right)^{2}$ идеал алгебры $L$, являющийся линейной оболочкой элементов вида $\left(u_{1} u_{2}\right)\left(u_{3} u_{4}\right), u_{i} \in L, i=1, \ldots, 4$.

Договоримся в градуированных тождественных соотношениях четные образующие обозначать через $x$ с индексами или без индексов, нечетные обозначать через $y$, а в случае любой четности - через $z$.

В многообразии $\mathbf{V}_{0}$ в силу одномерности пространства четных элементов выполняется следующее градуированное тождественное соотношение, а также его следствие:

$$
x_{1} x_{2} \equiv 0, \quad x y_{1} y_{2} \equiv x y_{2} y_{1} .
$$

Поскольку пространство нечетных элементов двумерно, то альтернирование по любым трем нечетным элементам будет также равно нулю.

ЛЕмма 1. В многообразии $\mathbf{V}_{0}$ выполняется тождество

$$
x z_{1}^{2} z_{2}^{2} \equiv x z_{2}^{2} z_{1}^{2}
$$

ДоКАЗАТЕЛЬСтво. Если $z_{1}$ и (или) $z_{2}$ являются четными, то (4) следует из (3). Пусть $x=h, z_{1}=\alpha e+\beta f, z_{2}=\gamma e+\delta f$. Тогда

$$
\begin{aligned}
x z_{1}^{2} z_{2}^{2} & =h(\alpha e+\beta f)^{2}(\gamma e+\delta f)^{2}=(2 \alpha e-2 \beta f)(\alpha e+\beta f)(\gamma e+\delta f)^{2} \\
& =(2 \alpha \beta h+2 \beta \alpha h)(\gamma e+\delta f)^{2}=4 \alpha \beta h(\gamma e+\delta f)^{2}=16 \alpha \beta \gamma \delta h .
\end{aligned}
$$

В силу симметричности выражения относительно коэффициентов получаем требуемое.

Из леммы 1 следует соотношение

$$
x z_{1} z_{2} z_{3} z_{4} \equiv x z_{3} z_{4} z_{1} z_{2}
$$

которое выполняется при условии, что переменные в парах $\left(z_{1}, z_{2}\right)$ и $\left(z_{3}, z_{4}\right)$ имеют одинаковую четность.

Докажем также следующее требуемое в дальнейшем соотношение:

$$
h e h f-h f h e=-8 h .
$$

Действительно, $h e h f-h f h e=2 e h f+2 f h e=-4 e f+4 f e=-8 h$.

Выпишем еще одно соотношение, следующее из доказательства леммы 1:

$$
h(e+f)^{2}=4 h .
$$

Структура строения полилинейной части многообразия, порожденного алгеброй $\operatorname{sl}(2, \Phi)$ без градуировки, полностью описана в работе [10]. В частности, 
доказано, что все кратности $m_{\lambda}$ кохарактеров в соответствующей сумме $(1)$ меньше или равны 1 . Отсюда следует, что это же свойство выполняется и для характера $\chi\left(P_{0, n}\right)$, т. е. в разложении

$$
\chi\left(P_{0, n}\right)=\sum_{\lambda \vdash n} m_{\lambda} \chi_{\lambda}
$$

все кратности $m_{\lambda}$ не превосходят единицы. Следует отметить, что полное описание строения пространств $P_{k, n-k}$ как модулей произведения $S_{k} \times S_{n-k}$ получено в работе [11].

Далее потребуется вид порождающего элемента неприводимого модуля в $P_{0, n}$, соответствующего разбиению $\lambda=(q+r, q), 2 q+r=n$, в случае, когда $q=2 k+1$. Отметим, что в случае четных $q$ и $r$ соответствующая кратность равна нулю.

Пусть $g_{0}\left(y_{1}, y_{2}\right)=y_{1} y_{2}$; определим оператор $d\left(y_{1}, y_{2}\right)$, где $y_{1}, y_{2} \in L_{1}$. Напомним, что $\operatorname{ad} z: w \mapsto w z$. Пусть $Y_{1}=\operatorname{ad} y_{1}, Y_{2}=\operatorname{ad} y_{2}$; тогда

$$
d\left(y_{1}, y_{2}\right)=Y_{1} Y_{1} Y_{2} Y_{2}-Y_{1} Y_{2} Y_{1} Y_{2}-Y_{2} Y_{1} Y_{2} Y_{1}+Y_{2} Y_{2} Y_{1} Y_{1},
$$

а также $g_{k}\left(y_{1}, y_{2}\right)=g_{k-1}\left(y_{1}, y_{2}\right) d\left(y_{1}, y_{2}\right), k=1,2, \ldots$. Заметим, что $d(y, y)=0$.

Докажем, что $g_{k} y_{1}^{r} \not \equiv 0$ для любых $k, r$. Подставим $y_{1}=e+f, y_{2}=f$ и получим $g_{0}(e+f, f)=h$, следовательно (см. (6)), $g_{k}(e+f, f)=g_{k}(e, f)=$ $(-8)^{k} h$. Тогда

$$
g_{k}(e+f, f)(e+f)^{r}=(-8)^{k} h(e+f)^{r}=a,
$$

где $a=(-8)^{k} 4^{s} h \neq 0$ при $r=2 s$ и $a=(-8)^{k} 4^{s} h(e+f) \neq 0$ при $r=2 s+1$.

ЗАмечАниЕ 2. Модуль, порожденный в $P_{0, n}, n=2(2 k+1)+r$, линеаризацией элемента $g_{k}\left(y_{1}, y_{2}\right) y_{1}^{r}$, соответствует разбиению $\lambda=(2 k+1+r, 2 k+1)$ и имеет степень $2(2 k+1)+r$.

Для доказательства первого условия критерия полиномиальности любого подмногообразия многообразия $\mathbf{V}_{0}$ потребуются несколько лемм.

ЛЕмма 2. В любом собственном подмногообразии $\mathbf{V}$ многообразия $\mathbf{V}_{0}$ въполняется тождество $g_{m}\left(y_{1}, y_{2}\right) y_{1}^{2 s} \equiv 0$.

ДокАЗАТЕЛЬСтво. Пусть в собственном подмногообразии выполняется полилинейное тождество $f\left(x_{1}, \ldots, x_{k}, y_{1}, \ldots, y_{n-k}\right) \equiv 0$, которое не выполняется в градуированной алгебре $\mathrm{sl}^{\mathrm{gr}}(2, \Phi)$. Подставим вместо четных образующих коммутаторы нечетных и получим тождество с аналогичным свойством, но только от нечетных порождающих. Поэтому сразу можно считать, что тождество имеет вид $f\left(y_{1}, \ldots, y_{n}\right) \equiv 0$.

Поскольку кохарактер $\chi\left(P_{0, n}\right)$ "лежит в полосе шириной, равной двум", а все кратности не превосходят 1 , то можно дополнительно считать, что рассматриваемое тождество соответствует разбиению $\lambda=(q+r, q)$. Покажем, что можно дополнительно считать, что $q$ нечетно. Предположим, что $q$ четно, тогда $r$ является нечетным, иначе в силу [10, теорема 4.2] это тождество выполнялось бы в самой алгебре $\mathrm{sl}(2, \Phi)$. Рассмотрим элемент $f\left(y_{1}, \ldots, y_{n}\right) y_{n+1}$ и породим им $S_{n+1}$-модуль в пространстве $P_{0, n+1}$. Заметим, что он по-прежнему не является градуированным тождеством алгебры $\mathrm{sl}^{\mathrm{gr}}(2, \Phi)$. Из правила РичардсонаЛиттлвуда и условия "расположения в полосе высотой, равной двум", учитывая, что в разбиении $\mu=(q+r+1, q)$ обе строки имеют четную длину 
(такие элементы задают тождества алгебры $\mathrm{sl}(2, \Phi)$ ), получаем, что построенный ненулевой модуль является неприводимым и соответствует разбиению $\lambda=((q+1)+(r-1), q+1)$, где $q+1-$ нечетное число. Таким образом, тождество, выполняющееся в собственном подмногообразии, соответствует разбиению $\lambda=(q+r, q)$ с нечетным $q$. Ранее было показано, что тождество $g_{m}\left(y_{1}, y_{2}\right) y_{1}^{r} \equiv 0$ при $q=2 m+1$ также соответствует разбиению $\lambda$. Поскольку кратность $m_{\lambda}$ в (1) равна 1 для $\mathrm{sl}(2, \Phi)$, мы получаем, что $g_{m}\left(y_{1}, y_{2}\right) y_{1}^{r} \equiv 0-$ тождество многообразия $\mathbf{V}$. Поскольку домножение этого тождества на $y_{1}$ снова дает нетривиальное тождество многообразия $\mathbf{V}$, мы можем считать $r$ четным. Лемма доказана.

В полученном тождественном соотношении можно “разделить" переменные, т. е. получить более сильное тождество.

ЛЕмма 3. В любом собственном подмногообразии $\mathbf{V} \subset \operatorname{var}^{\mathrm{gr}}(\mathrm{sl}(2, \Phi))$ выполняется тождество вида

$$
g_{m}\left(y_{1}, y_{2}\right) y_{3}^{2 s} \equiv 0
$$

ДокАЗАтЕльство. Пусть $\bar{g}_{m}$ - полная линеаризация полинома $g_{m}$. Заметим, что степень полинома $\bar{g}_{m}$ есть $t=2(2 m+1)$ и он порождает неприводимый модуль, соответствующий разбиению $\lambda=(2 m+1,2 m+1)$. Полная линеаризация $g_{m}\left(y_{1}, y_{2}\right) y_{3}^{2 s}$ имеет вид

$$
A=\sum_{p \in S_{2 s}} \bar{g}_{m}\left(y_{2 s+1}, \ldots, y_{n}\right) y_{p(1)} \cdots y_{p(2 s)},
$$

где $n=t+2 s$.

$\left(S_{t} \times S_{2 s}\right)$-модуль, порожденный элементом $A$, является тензорным произведением модулей, соответствующих разбиениям $\lambda=(2 m+1,2 m+1)$ и $\mu=(2 s)$. По правилу Ричардсона-Литтлвуда неприводимые $S_{n}$-подмодули в разложении $\Phi S_{n} A$ соответствуют только разбиению $(2 m+1+2 s, 2 m+1)$ и разбиениям на три или более части, которые являются нулевыми, так как кохарактер "лежит в полосе шириной, равной двум". В завершение доказательства отметим, что неприводимый подмодуль, соответствующий разбиению $(2 m+1+2 s, 2 m+1)$, порождается элементом $g_{m}\left(y_{1}, y_{2}\right) y_{1}^{2 s}$. Но этот модуль должен иметь нулевую кратность в любом собственном подмногообразии, поскольку в исходном многообразии $\operatorname{var}^{\mathrm{gr}}(\operatorname{sl}(2, \Phi))$ его кратность равна единице.

ПрЕДЛОЖЕНИЕ 3. Пусть V - собственное подмногообразие многообразия $\mathbf{V}_{0}$. Тогда существует такое число $M$, что для любого разбиения $\lambda=$ $\left(\lambda_{1}, \lambda_{2}\right)$ при условии $\lambda_{2}>M$ соответствующий элемент является тождеством в $\mathbf{V}$ на нечетных переменных.

ДокАЗАТЕльство. На первом шаге получим тождество вида $g_{m}\left(y_{1}, y_{2}\right) \equiv 0$. По лемме 3 в подмногообразии $\mathbf{V}$ выполняется тождество $g_{m}\left(y_{1}, y_{2}\right) y_{3}^{2 s} \equiv 0$. Проводя частичную линеаризацию и учитывая условия $(3),(5)$, получим следствие $g_{m}\left(y_{1}, y_{2}\right) y_{4} y_{3}^{2 s-1} \equiv 0$. Аналогично предыдущей частичной линеаризации проведем частичную линеаризацию полученного элемента, в результате получим

$$
g_{m}\left(y_{1}, y_{2}\right) y_{4} y_{5} y_{3}^{2 s-2}+(2 s-2) g_{m}\left(y_{1}, y_{2}\right) y_{4} y_{3} y_{5} y_{3}^{2 s-3} \equiv 0 .
$$

Используя (3), (5), из тождества (9) получаем следствие:

$$
g_{m}\left(y_{1}, y_{2}\right) y_{4} y_{5} u v y_{3}^{2 s-2}+(2 s-2) g_{m}\left(y_{1}, y_{2}\right) y_{4} y_{3} u v y_{5} y_{3}^{2 s-3} \equiv 0,
$$

где $u, v$ - также нечетные переменные. 
Альтернируя левую часть (10) по переменным $y_{4}, u$ и по переменным $y_{5}, v$, подставляя $y_{4}=y_{5}=y_{1}, u=v=y_{2}$ и учитывая (3) и (5), получаем следствие:

$$
\begin{aligned}
w^{\prime} & =g_{m} \bar{y}_{1} \tilde{y}_{1} \bar{y}_{2} \tilde{y}_{2} y_{3}^{2 s-2}+(2 s-2) g_{m} y_{3} \tilde{y}_{1} \tilde{y}_{2} \bar{y}_{2} \bar{y}_{1} y_{3}^{2 s-3} \\
& =g_{m} \bar{y}_{1} \tilde{y}_{1} \bar{y}_{2} \tilde{y}_{2} y_{3}^{2 s-2}-(2 s-2) g_{m} y_{3} \tilde{y}_{1} \tilde{y}_{2} \bar{y}_{1} \bar{y}_{2} y_{3}^{2 s-3} \\
& =g_{m} d\left(y_{1}, y_{2}\right) y_{3}^{2 s-2}-(2 s-2) g_{m} y_{3}\left(y_{1} y_{2}\right)\left(y_{1} y_{2}\right) y_{3}^{2 s-3} .
\end{aligned}
$$

Здесь мы используем соглашение о том, что одинаковые символы над переменными (черточка, волна) означают, что по ним проведено альтернирование. Например, $a \bar{y}_{1} y_{2} \bar{y}_{3}=a y_{1} y_{2} y_{3}-a y_{3} y_{2} y_{1}$, a

$$
a \tilde{y}_{1} \tilde{y}_{2} \tilde{y}_{3}=\sum_{\sigma \in S_{3}}(-1)^{\sigma} a y_{\sigma(1)} y_{\sigma(2)} y_{\sigma(3)} .
$$

Покажем, что элемент $w^{\prime}$ не равен нулю в многообразии $\operatorname{var}^{\mathrm{gr}}(\mathrm{sl}(2, \Phi))$. Подставим $y_{1}=e+f, y_{2}=f, y_{3}=e+f$ и получим

$$
\begin{array}{rl}
(-8)^{m+1} & h(e+f)^{2 s-2}-(2 s-2)(-8)^{m} h(e+f) h h(e+f)^{2 s-3} \\
& =(-8)^{m+1} h(e+f)^{2 s-2}-(2 s-2)(-8)^{m} h((e+f) h h)(e+f)^{2 s-3} \\
& =(-8)^{m+1} h(e+f)^{2 s-2}-(2 s-2)(-8)^{m} h(4(e+f))(e+f)^{2 s-3} \\
& =(-8)^{m}\left(-8 h(e+f)^{2 s-2}-4(2 s-2) h(e+f)^{2 s-2}\right) \\
& =(-8)^{m+1} \operatorname{sh}(e+f)^{2 s-2} \neq 0 .
\end{array}
$$

Итак,

$$
g_{m+1} y_{3}^{2 s-2}-(2 s-2) g_{m} y_{3}\left(y_{1} y_{2}\right)\left(y_{1} y_{2}\right) y_{3}^{2 s-3} \equiv 0
$$

- нетривиальное тождество в V. Кроме того, так как мы подставляли $e+f$ вместо $y_{1}$ и $y_{3}$, то нетривиальным будет также тождество

$$
u\left(y_{1}, y_{2}\right)=g_{m+1} y_{1}^{2 s-2}-(2 s-2) g_{m} y_{1}\left(y_{1} y_{2}\right)\left(y_{1} y_{2}\right) y_{1}^{2 s-3} \equiv 0 .
$$

Отметим, что степень тождества $u\left(y_{1}, y_{2}\right) \equiv 0$ равна $2(2 m+3)+2 s-2$, причем степень по $y_{1}$ равна $2 m+2 s+1$, а по $y_{2}$ равна $2 m+3$. По тем же причинам, что и для $g_{k} y_{1}^{r}$ (см. замечание 2$)$, в разложении $S_{n}$-модуля, порожденного линеаризацией элемента $u\left(y_{1}, y_{2}\right)$, нет слагаемых, отличных от неприводимого модуля, соответствующего разбиению $\lambda=(2(m+1)+1+(2 s-2), 2 s-2)$. Следовательно, $g_{m+1} y_{3}^{2 s-2} \equiv 0$ является нетривиальным тождеством многообразия $\mathbf{V}$.

Аналогичным образом понижая степень по переменной $y_{3}$, получаем тождество $g_{m+s} \equiv 0$, а следовательно, и любое тождество вида $g_{k} y_{3}^{r} \equiv 0$, в котором $k \geqslant m+s, r \geqslant 0$. Поскольку именно элемент $g_{k}\left(y_{1}, y_{2}\right) y_{1}^{r}$ соответствует разбиению $\lambda=\left(\lambda_{1}, \lambda_{2}\right)$, где $\lambda_{1}=(2 k+1)+r, \lambda_{2}=2 k+1$, то в случае нечетной длины второй строки соответствующей диаграммы Юнга предложение доказано.

Покажем, что в случае четной длины, большей $M=2(m+s)+1$, также получаем требуемое. Пусть разбиение $\lambda=\left(\lambda_{1}, \lambda_{2}\right)$ - такое, что $\lambda_{2}>M$, причем $\lambda_{2}$ является четным. По доказанному элемент, соответствующий разбиению $\left(\lambda_{1}-1, \lambda_{2}-1\right)$ с нечетной длиной второй строки, является нетривиальным тождеством в $\mathbf{V}$. Если умножить его на произведение $y_{1} y_{2}$, то полученный элемент по-прежнему будет нетривиальным тождеством многообразия $\mathbf{V}$, а кроме того, будет соответствовать разбиению $\lambda=\left(\lambda_{1}, \lambda_{2}\right)$. 
ЗАМЕЧАНИЕ 3. В качестве следствия отметим, что для любого подмногообразия $\mathbf{V}$ многообразия $\mathbf{V}_{0}$ существует такое число $M$, что в многообразии $\mathbf{V}$ выполняется любое полилинейное тождество от нечетных образующих, если каждый из одночленов является произведением элементов, не менее $M$ из которых представляют собой коммутаторы нечетных образующих. Действительно, такой элемент имеет не менее $M$ кососимметрических пар переменных, а следовательно, порождает модуль симметрической группы, в разложении которого на неприводимые компоненты присутствуют лишь слагаемые, соответствующие диаграммам со второй строкой, имеющей длину, большую или равную $M$. По предложению 3 такие слагаемые равны нулю, значит, и сам модуль равен нулю, а порождающий его элемент является тождеством многообразия $\mathbf{V}$.

ПреДЛОЖЕНИЕ 4. Для многообразия $\mathbf{V}$ существует такое число $T$, что для любого $t \geqslant T$ в $\mathbf{V}$ выполняется обычное неградуированное тождество

$$
\left(\left(z_{11} z_{12}\right)\left(z_{13} z_{14}\right)\right) \cdots\left(\left(z_{t 1} z_{t 2}\right)\left(z_{t 3} z_{t 4}\right)\right) \equiv 0
$$

ДокАЗАтЕльство. Каждый фрагмент вида $\left(\left(z_{i 1} z_{i 2}\right)\left(z_{i 3} z_{i 4}\right)\right)$ при подстановке в него более двух четных образующих или двух четных образующих в одну скобку будет равен нулю в силу одномерности пространства $(\operatorname{sl}(2, \Phi))_{0}$. При подстановке двух четных и двух нечетных образующих $\left(\left(y_{1} x_{1}\right)\left(y_{2} x_{2}\right)\right)$ получаем коммутатор двух нечетных элементов. При подстановке только одной четной переменной мы получаем произведение вида $\left(y_{1} y_{2}\right)\left(y_{3} x_{1}\right)$, в котором сомножитель $y_{1} y_{2}$ является коммутатором нечетных элементов. В случае подстановки всех нечетных образующих получаем даже два коммутатора нечетных элементов. Таким образом, при любой подстановке выполнено тождество (11) в силу замечания 3 .

ПРЕДЛОЖЕНИЕ 5. Пусть V - собственное подмногообразие многообразия $\mathbf{V}_{0}$. Тогда $\mathbf{V} \subset \mathbf{N}_{c} \mathbf{A}$ для некоторого с.

ДокАЗАТЕЛЬСтво. Из предложения 4 следует, что многообразие $\mathbf{V}$ является разрешимым, поэтому оно является собственным подмногообразием многообразия алгебр Ли, порожденного алгеброй $\mathrm{sl}(2, \Phi)$. Многообразие $\operatorname{var}(\operatorname{sl}(2, \Phi))$ подробно исследовано в работах [10], [12], [13]. В частности, известно, что любое его собственное подмногообразие состоит из алгебр с нильпотентным коммутантом, т. е. в нем для некоторого $c$ выполняется тождество

$$
\left(z_{11} z_{12}\right)\left(z_{21} z_{22}\right) \cdots\left(z_{c+1,1} z_{c+1,2}\right) \equiv 0
$$

и, следовательно, выполняется включение $\mathbf{V} \subset \mathbf{N}_{c} \mathbf{A}$.

Следующее предложение связано со вторым условием теоремы 1 только относительно градуированной алгебры $\mathrm{sl}^{\mathrm{gr}}(2, \Phi)$.

ПРЕДЛОЖЕНИЕ 6. Пусть V - собственное подмногообразие многообразия $\mathbf{V}_{0}$. Тогда существует такое число $m$, что любой полилинейный полином, содержащий не менее $m$ четных и $m$ нечетных образующих, тождественно равен нулю в $\mathbf{V}$.

ДоКАЗАТЕЛЬСТво. Согласно предложению 5 в многообразии выполняется обычное тождество (12). Напомним, что из соображений размерности четной и нечетной компонент в $\mathbf{V}$ выполняется тождество $x_{1} x_{2} \equiv 0$ (см. (3)), кроме того, любое выражение, альтернированное по трем нечетным образующим, 
также является тождеством. Докажем, что в этом случае выполняются также условия

$$
\begin{gathered}
y_{0} x_{1} \cdots x_{m} y_{1} \cdots y_{k} \in\left(L^{2}\right)^{2}, \quad m \geqslant 2, \quad k \geqslant 1, \\
\left(y_{10} x_{10} y_{11} \cdots y_{1 m}\right)\left(y_{20} x_{21} \cdots x_{2 k}\right) \in\left(L^{2}\right)^{3}, \quad m \geqslant 2, \quad k \geqslant 2 .
\end{gathered}
$$

По модулю $\left(L^{2}\right)^{2}$ произведение $y_{0} x_{1} \cdots x_{m} y_{1}$ сравнимо с $y_{0} x_{1} \cdots x_{m-1} y_{1} x_{m}$, а последнее равно нулю, так как $y_{0} x_{1} \cdots x_{m-1} y_{1}-$ четный элемент. Отсюда следует условие (13).

Докажем выполнение условия (14). Пусть сначала $m$ является четным числом. Тогда

$$
\begin{aligned}
& \left(y_{10} x_{10} y_{11} \cdots y_{1 m}\right)\left(y_{20} x_{21} \cdots x_{2 k}\right) \\
& =\left(y_{10} x_{10} y_{11} \cdots y_{1 m}\right)\left(y_{20} x_{21} \cdots x_{2, k-1}\right) x_{2 k} \\
& \quad-\left(y_{10} x_{10} y_{11} \cdots y_{1 m} x_{2 k}\right)\left(y_{20} x_{21} \cdots x_{2, k-1}\right) .
\end{aligned}
$$

Первое слагаемое равно нулю в силу (3), а для второго слагаемого в силу того, что по модулю $\left(L^{2}\right)^{2}$ выполняется равенство $y_{10} x_{10} y_{11} \cdots y_{1 m} x_{2 k}=$ $y_{10} x_{10} x_{2 k} y_{11} \cdots y_{1 m}$, можно применить условие (13). В итоге получим требуемое.

Пусть теперь $m$ является нечетным числом. Тогда

$$
\begin{aligned}
\left(y_{10} x_{10} y_{11} \cdots y_{1 m}\right)\left(y_{20} x_{21} \cdots x_{2 k}\right) & \\
= & -\left(y_{10} x_{10} y_{11} \cdots y_{1, m-1}\right)\left(y_{20} x_{21} \cdots x_{2 k}\right) y_{1 m} \\
& \quad+\left(y_{10} x_{10} y_{11} \cdots y_{1, m-1}\right)\left(y_{20} x_{21} \cdots x_{2 k} y_{1 m}\right) .
\end{aligned}
$$

Первое слагаемое принадлежит $\left(L^{2}\right)^{3}$, так как

$$
\left(y_{10} x_{10} y_{11} \cdots y_{1, m-1}\right)\left(y_{20} x_{21} \cdots x_{2 k}\right)
$$

принадлежит $\left(L^{2}\right)^{3}$ по доказанному выше, поскольку $m-1$ является четным числом. Для второго слагаемого сомножитель $y_{20} x_{21} \cdots x_{2 k} y_{1 m}$ принадлежит $\left(L^{2}\right)^{2}$ по условию (13). Выполнение условия (14) полностью доказано.

Индукцией по $c$ из тождества (12), которое выполняется в рассматриваемом многообразии, покажем теперь, что существует такое $M_{c}$, что выполняется любое тождество вида $f\left(x_{1}, \ldots, x_{m}, y_{1}, \ldots, y_{k}\right) \equiv 0$ при условии $m, k \geqslant M_{c}$.

Если $c=1$, то из условия (13) вытекает, что достаточно задать $M_{1}=2$. Рассмотрим отдельно два случая: $c=2$ и $c>2$.

Пусть $c=2$. Положим $M_{2}=6$. Действительно, так как $M_{2}>M_{1}$, из предыдущего шага следует, что $f\left(x_{1}, \ldots, x_{m}, y_{1}, \ldots, y_{k}\right)$ принадлежит $\left(L^{2}\right)^{2}$, т. е. является линейной комбинацией произведений коммутаторов. Рассмотрим одно из слагаемых, коммутаторы обозначим $a, b$. Заметим, что по модулю $\left(L^{2}\right)^{3}$ в элементах $a, b$ можно упорядочить четные и нечетные переменные, т. е. можно считать, что коммутаторы имеют вид

$$
y_{1} \cdots y_{k}
$$

или

$$
y_{1} x_{1} \cdots x_{r} y_{2} \cdots y_{k}
$$

Если в одном из коммутаторов количества четных и нечетных переменных одновременно не меньше двух, то по доказанному этот коммутатор принадлежит $\left(L^{2}\right)^{2}$, а поэтому $a b \in\left(L^{2}\right)^{3}$. В противном случае один из коммутаторов содержит не меньше пяти нечетных переменных (пусть это будет $a$ ), а другой не меньше пяти четных переменных, причем обязательно (иначе в силу (3) он будет равен нулю) содержит ровно одну нечетную переменную, 
т. е. $b=y_{21} x_{21} \cdots x_{2 r}$, где $r \geqslant 5$. Если $a$ состоит только из нечетных переменных: $a=y_{21} \cdots y_{2 k}, k \geqslant 5$, то $a=-y_{23} x y_{24} \cdots y_{2 k}$, где $x=y_{21} y_{22}$ - четный элемент, и по (14) получаем требуемое. В противном случае элемент $a$ имеет вид (16), более точно, $a=y_{11} x_{11} y_{12} \cdots y_{1 k}, k \geqslant 5$, и можно сразу использовать условие (14).

Пусть теперь $c>2$, и предположим, что для $c-1$ и для некоторого $M_{c-1} \leqslant 6(c-1)$ доказано требуемое. Положим $M_{c}=6 c$. Тогда $M_{c}>M_{c-1}$ и по предположению индукции $f\left(x_{1}, \ldots, x_{m}, y_{1}, \ldots, y_{k}\right) \in\left(L^{2}\right)^{c}$, если $m, k \geqslant M_{c}$. Значит, $f$ можно выразить через произведения левонормированных коммутаторов $a_{1}, \ldots, a_{c}$, кроме того, по модулю $\left(L^{2}\right)^{c+1}$ в каждом из $a_{i}$ все переменные, начиная с третьей, можно переставлять. Заметим еще раз, что $a_{i}$ не может начинаться с двух четных переменных (оно будет равно нулю по (3)), поэтому $a_{i}=y_{i 1} y_{i 2} \cdots$, и в этом случае все переменные нечетные (иначе $a_{i}$ равно нулю по (3), так как произведение $y_{i 1} y_{i 2}$ является четной переменной) или $a_{i}=y_{i 0} x_{i 1} \cdots$.

Поскольку $M_{c}=6 c$, то возможны два случая. В первом существует коммутатор $a_{i}$, который содержит одновременно не меньше $M_{2}=6$ четных и нечетных переменных одновременно. В этом случае $a_{i} \in\left(L^{2}\right)^{2}$, а значит, всё произведение принадлежит $\left(L^{2}\right)^{c+1}$. Действительно, произведение можно выразить в виде линейной комбинации левонормированных произведений, в которых коммутатор $a_{i}$ расположен на первом месте. Во втором случае, т. е. если такого коммутатора нет, существует коммутатор $a_{i}$, в котором не меньше шести четных переменных, т. е. $a_{i}=x y x_{1} x_{2} x_{3} \cdots$, и другой коммутатор $a_{j}$, состоящий из не менее семи нечетных переменных и не более одной четной. Можно считать, что этот коммутатор имеет вид $a_{j}=x^{\prime} y_{1} y_{2} y_{3} y_{4} y_{5} \cdots$, где $x^{\prime}$ - четная переменная. Напомним, что результат альтернирования по любым трем нечетным переменным равен нулю. Применим альтернирование к $u_{1}=x y, u_{2}=y_{5}, u_{3}=x^{\prime} y_{1} y_{2} y_{3}$. Получим, что исходный элемент равен сумме пяти слагаемых, каждое из которых принадлежит $\left(L^{2}\right)^{c+1}$. Действительно, это очевидно в случае замены $u_{2}$ на $u_{1}$ или $u_{3}$, а слагаемое, которое получается перестановкой $u_{1}$ и $u_{3}$, приводит к появлению коммутатора вида $x^{\prime} y_{1} y_{2} y_{3} x_{1} x_{2} x_{3} \cdots$, который по (13) приводит к появлению $(c+1)$-го коммутатора, так как принадлежит $\left(L^{2}\right)^{2}$.

Teорема 2. Пусть $\mathbf{U} \subset \operatorname{var}\left(G\left(\mathrm{sl}^{\mathrm{gr}}(2, \Phi)\right)\right)$ - собственное подмногообразие. Тогда $\mathbf{U}$ имеет полиномиальный рост.

ДоКАЗАТЕЛЬСТВо. По предложению 1 существует собственное подмногообразие $\mathbf{V}$ многообразия $\operatorname{var}^{\mathrm{gr}}\left(\mathrm{sl}^{\mathrm{gr}}(2, \Phi)\right)$, для которого $\Theta(\mathbf{V})=\mathbf{U}$.

По предложению 5 в многообразии $\mathbf{V}$ выполняется тождество (12). Рассмотрим относительно свободную алгебру $L \in \mathbf{V}$ со счетными множествами четных $x_{1}, x_{2}, \ldots$ и нечетных $y_{1}, y_{2}, \ldots$ образующих. Как обычная алгебра Ли, алгебра $L$ по предложению 5 имеет нильпотентный коммутант, т. е. $L \in \mathbf{N}_{c} \mathbf{A}$, поэтому многообразие $\operatorname{var}(L)$ имеет полиномиальный рост [10]. Кроме того, по предложению 6 в многообразии $\mathbf{V}$ тождественно равно нулю любое выражение, содержащее не менее $m$ четных и нечетных образующих одновременно. Отсюда по предложению 2 многообразие $\mathbf{U}$ имеет полиномиальный рост, что и завершает доказательство теоремы.

Теорема 3. Для многообразия $\operatorname{var}\left(G\left(\mathrm{sl}^{\mathrm{gr}}(2, \Phi)\right)\right)$ выполнено равенство

$$
\operatorname{Exp}\left(\operatorname{var}\left(G\left(\operatorname{sl}^{\text {gr }}(2, \Phi)\right)\right)\right)=3 .
$$

ДокАЗАТЕЛЬСтво. Сначала получим нижнюю оценку. Обозначим через $P_{m, 4 k+2}$ пространство полилинейных лиевских многочленов от $m$ четных 
и $4 k+2$ нечетных переменных в свободной $\mathbb{Z}_{2}$-градуированной алгебре. Сначала покажем, что в $P_{m, 4 k+2}$ можно выбрать элемент специального вида, не равный тождественно нулю в алгебре $\mathrm{sl}^{\mathrm{gr}}(2, \Phi)$.

Ранее был построен элемент $g_{k}(x, y)$ степени $4 k+2$, линеаризация $f_{0}\left(y_{1}\right.$, $\left.y_{2}, \ldots, y_{4 k+2}\right)$ которого порождала неприводимый $S_{4 k+2}$-модуль, соответствующий разбиению $\lambda=(2 k+1,2 k+1)$, т. е. диаграмме Юнга, состоящей из двух строк длиной $2 k+1$. При этом все переменные $y_{1}, y_{2}, \ldots, y_{4 k+2}$ являются нечетными, а $f_{0}$ может принимать в алгебре $\mathrm{sl}^{\mathrm{gr}}(2, \Phi)$ ненулевые значения, кратные $h$. Отсюда следует, что для любого $m$ не равен тождественно нулю также элемент $f_{0}\left(y_{1}, y_{2}, \ldots, y_{4 k+2}\right)\left(y_{0} x^{m}\right)$. В грассмановой оболочке будет не равен тождественно нулю элемент, построенный по диаграмме Юнга из $4 k+3+m$ клеток, содержащей строку длиной не меньше $m$ и два столбца высотой не меньше $2 k+1$ одновременно. Из формулы крюков при $m=2 k+1$ получаем, что коразмерность удовлетворяет неравенству $c_{n}\left(\mathrm{sl}^{\mathrm{gr}}(2, \Phi)\right) \geqslant \frac{3^{n}}{n^{6}}$.

Получим верхнюю оценку. Рассмотрим одночлены степени $n$ с некоторой фиксированной расстановкой скобок от переменных $z_{1}, z_{2}, \ldots, z_{n}$. Их линейная оболочка является $S_{n}$-модулем. Обозначим через $Q_{n}$ фактормодуль построенного модуля по тождествам супералгебры $G\left(\mathrm{sl}^{\mathrm{gr}}(2, \Phi)\right)$. Рассмотрим его характер

$$
\chi\left(Q_{n}\right)=\sum_{\lambda \vdash n} m_{\lambda} \chi_{\lambda} .
$$

Покажем сначала, что $m_{\lambda}=0$, если диаграмма $\lambda$ не лежит в бесконечном крюке $H(1,2)$ из одной строки и двух столбцов.

Предположим, что $m_{\psi} \neq 0$ и $\psi$ не лежит в крюке $H(1,2)$. Это означает, что найдется соответствующий полином $f^{\prime}\left(z_{1}, z_{2}, \ldots, z_{n}\right)$, не равный тождественно нулю в алгебре $G\left(\mathrm{sl}^{\mathrm{gr}}(2, \Phi)\right)$. Таким образом, можно считать, что существует подстановка четных переменных вместо переменных $z_{1}, \ldots, z_{k}$ и нечетных переменных вместо $z_{k+1}, \ldots, z_{n}$, при которой $f^{\prime}$ не равно нулю. Тогда $f=f\left(x_{1}, \ldots, x_{k}, y_{1}, \ldots, y_{n-k}\right)$ не является градуированным тождеством алгебры sl ${ }^{\mathrm{gr}}(2, \Phi)$, где $f=\Theta f^{\prime}$.

Породим этим элементом $\left(S_{k} \times S_{n-k}\right)$-модуль $M$. Пусть $\varphi-$ характер одного из неприводимых подмодулей модуля $M$. Тогда, как известно (см., например, [14]), выполняется равенство

$$
\langle\operatorname{Res} \psi, \varphi\rangle=\langle\operatorname{Ind} \varphi, \psi\rangle
$$

и $\varphi$ соответствует паре диаграмм Юнга $(\mu, \nu), \mu \vdash k, \nu \vdash n-k$. Поскольку размерность четной компоненты алгебры Ли $\mathrm{sl}^{\mathrm{gr}}(2, \Phi)$ равна единице, а нечетной - двум, нетрудно показать, что $\mu \subset H(1,0), \nu \subset H(0,2)$, т. е. диаграмма $\mu$ имеет одну строку, а $\nu$ имеет не более двух столбцов. Но тогда из правила Ричардсона-Литтлвуда вытекает, что индуцированный с $\varphi$ характер Ind $\varphi$ группы $S_{n}$ лежит в крюке $H(1,2)$, поэтому кратность $\psi$ в Ind $\varphi$ равна нулю.

Другими словами, правая часть (18) равна нулю, а левая часть $\langle\operatorname{Res} \psi, \varphi\rangle$ отлична от нуля. Полученное противоречие означает, что предположение $f \not \equiv 0$ ложно, т. е. $\psi \subset H(1,2)$ в $(17)$.

Из формулы крюков для размерности неприводимого $S_{n}$-представления легко получить следующее ограничение для любого $\chi_{\lambda} \subset H(1,2)$ :

$$
\chi_{\lambda}(1) \leqslant 3^{n} n^{2} .
$$

Теперь покажем, что различные расстановки скобок, а также кратности характера $\chi_{\lambda}$ при фиксированной расстановке дают лишь полиномиальные множители в оценке сверху. 
Зафиксируем разбиение $\lambda \vdash n, \lambda \subset H(1,2)$. Пусть $r$ - длина первой строки, $s+1$ - высота первого столбца, а $t+1$ - высота второго столбца. Напомним, что $Q_{n}$ - пространство полилинейных неассоциативных элементов с некоторой фиксированной расстановкой скобок, профакторизованное по тождествам супералгебры $G\left(\mathrm{sl}^{\mathrm{gr}}(2, \Phi)\right)$. Пусть $W_{1} \oplus \cdots \oplus W_{m}$ - сумма неприводимых $S_{n}$-модулей в $Q_{n}$ с характером $\chi_{\lambda}$. Хорошо известно (см., например, [15]), что можно выбрать порождающие полиномы $f_{i} \in W_{i}, i=1, \ldots, m$, таким образом, чтобы каждый $f_{i}\left(z_{1}, \ldots, z_{n}\right)$ был симметричен по $r$ переменным $z_{1}, \ldots, z_{r}$, кососимметричен по $s$ переменным $z_{r+1}, \ldots, z_{r+s}$ и кососимметричен по $t$ переменным $z_{r+s+1}, \ldots, z_{n}$. Аналогичным образом можно построить полиномы в пространстве с другими скобочными структурами. Наша ближайшая цель доказать, что общее количество линейно независимых полиномов такого типа ограничено полиномиальной функцией.

Докажем, что $m<48(n+1)^{2}+1$. Предположим противное: пусть $m \geqslant$ $48(n+1)^{2}+1$. Рассмотрим линейную комбинацию $\delta_{1} f_{1}+\cdots+\delta_{m} f_{m}$ с неопределенными коэффициентами $\delta_{1}, \ldots, \delta_{m}$ и докажем, что существуют такие элементы $\delta_{1}, \ldots, \delta_{m}$ поля $\Phi$, что в многообразии $\operatorname{var}\left(G\left(\mathrm{sl}^{\mathrm{gr}}(2, \Phi)\right)\right)$ выполнено тождество $\delta_{1} f_{1}+\cdots+\delta_{m} f_{m} \equiv 0$. Определим тип произвольной подстановки $\varphi: z_{i} \mapsto a_{i} \otimes g_{i}$, где $a_{i} \in \operatorname{sl}(2, \Phi)$ принимают значения базисных векторов $e, f, h \in \operatorname{sl}(2, \Phi)$, а $g_{i} \in G$. Типом такой подстановки назовем строку

$$
\left(\alpha_{1}, \alpha_{2}, \alpha_{3}, \beta_{1}, \beta_{2}, \beta_{3}, \gamma_{1}, \gamma_{2}, \gamma_{3}\right)
$$

в которой $\alpha_{1}-$ количество базисных векторов $e \in \operatorname{sl}(2, \Phi)$, встречающихся в наборе $a_{1}, \ldots, a_{r}$. Число $\alpha_{2}$ (соответственно, $\left.\alpha_{3}\right)$ - количество базисных векторов $f$ (соответственно, $h$ ) в том же наборе. Аналогично определяются тройки чисел $\beta_{1}, \beta_{2}, \beta_{3}$ и $\gamma_{1}, \gamma_{2}, \gamma_{3}$ только относительно наборов $a_{r+1}, \ldots, a_{r+s}$ и $a_{r+s+1}, \ldots, a_{n}$.

Поскольку $z_{1}, \ldots, z_{r}$ - симметричные, а $z_{r+1}, \ldots, z_{r+s}$ и $z_{r+s+1}, \ldots, z_{n}$ - кососимметричные переменные, то $\alpha_{1}, \alpha_{2}, \beta_{3}, \gamma_{3} \leqslant 1$. Кроме того, $\beta_{1}+\beta_{2}=s, s-1$, a $\gamma_{1}+\gamma_{2}=t, t-1$. Поэтому количество различных типов подстановок меньше $16(n+1)^{2}$.

Заметим теперь, что если $\varphi$ и $\psi$ - подстановки одинакового типа, то $\varphi\left(f_{i}\right)=$ $\pm \psi\left(f_{i}\right)$, причем знак одинаков для всех $f_{1}, \ldots, f_{m}$. Заметим также, что все значения имеют вид $\varphi\left(f_{i}\right)=\left(\mu_{i} e+\nu_{i} f\right) \otimes g_{1} \cdots g_{n}$ либо вид $\varphi\left(f_{i}\right)=\rho_{i} h \otimes g_{1} \cdots g_{n}$, в зависимости от четности $a_{j}, g_{j}, j=1, \ldots, n$.

Выберем для каждого из возможных $T<16(n+1)^{2}$ типов по одной подстановке $\varphi_{1}, \ldots, \varphi_{T}$. Тогда для каждой из них значение $\varphi_{j}\left(f_{i}\right)$ определяется тремя коэффициентами $\mu_{i j}, \nu_{i j}, \rho_{i j}$. Отсюда следует, что если $m>48(n+1)^{2}$, то существует нетривиальный набор скаляров $\delta_{1}, \ldots, \delta_{m}$ таких, что $\varphi_{j}\left(\delta_{1} f_{1}+\cdots\right.$ $\left.\cdots+\delta_{m} f_{m}\right)=0$ в алгебре $G\left(\mathrm{sl}^{\mathrm{gr}}(2, \Phi)\right)$ для всех $j=1, \ldots, T$. С учетом замечания о подстановках одинакового типа это и означает, что $\delta_{1} f_{1}+\cdots+\delta_{m} f_{m} \equiv 0-$ тождество в $G\left(\mathrm{sl}^{\mathrm{gr}}(2, \Phi)\right)$.

Доказанное ограничение на число изоморфных неприводимых слагаемых, а также (19) означают, что для заданного вида диаграммы Юнга $\lambda$ коразмерность не превышает $48(n+1)^{2} n^{2} 3^{n}$. Поскольку количество разбиений $\lambda \vdash n$, лежащих в крюке $H(1,2)$, ограничено сверху величиной $n^{3}$, получаем неравенство

$$
c_{n}\left(G\left(\mathrm{sl}^{\mathrm{gr}}(2, \Phi)\right)\right) \leqslant C \cdot 3^{n} n^{7},
$$

которое вместе с нижней оценкой позволяет завершить доказательство теоремы. 


\section{§ 4. Разрешимые многообразия почти полиномиального роста}

Уточним оценки для экспоненциального роста упомянутых в начале статьи двух известных ранее многообразий почти полиномиального роста и докажем, что их экспоненты в точности равны двум.

Теорема 4. Пусть $\mathbf{A}^{2}$ - многообразие метабелевых супералгебр Ли, определяемое тождеством $\left(z_{1} z_{2}\right)\left(z_{3} z_{4}\right) \equiv 0$. Тогда экспонента многообразия $\mathbf{A}^{2}$ равна двум, т.е. $\operatorname{Exp}\left(\mathbf{A}^{2}\right)=2$.

ДоказАТЕльство. Сначала получим оценку снизу. Поскольку произведение $x_{1} \cdots x_{m} y_{1} \cdots y_{k}$ не равно нулю в свободной метабелевой супералгебре Ли, где $x_{1}, \ldots, x_{m}$ - четные, а $y_{1}, \ldots, y_{k}$ - нечетные переменные, то полином

$$
\sum_{\sigma \in S_{m}} \sum_{\tau \in S_{k}}(-1)^{\tau} x_{0} x_{\sigma(1)} \cdots x_{\sigma(m)} y_{\tau(1)} \cdots y_{\tau(k)}
$$

не является тождеством и содержит симметрический набор из $m$ переменных и кососимметрический набор из $k$ переменных, где $n=m+k+1$. Таким образом, полилинейная часть метабелева многообразия как модуль симметрической группы $S_{n}$ содержит ненулевые неприводимые подмодули для $k=0, \ldots, n-1$, соответствующие диаграммам Юнга, которые включают поддиаграмму для разбиения $\left(m, 1^{k}\right)$ числа $n-1=m+k$. Используя формулу крюков, получаем, что $\gamma_{n}\left(\mathbf{A}^{2}\right)>2^{n}$.

Найдем теперь верхнюю оценку для размерности $\gamma_{n}\left(\mathbf{A}^{2}\right)$. Обозначим через $A=A_{0} \oplus A_{1}$ свободную супералгебру многообразия $\mathbf{A}^{2}$ с бесконечным числом четных и нечетных образующих, а через $B=G(B)=B_{0} \oplus B_{1}$ - её грассманову оболочку. Тогда $B$ - градуированная алгебра Ли. Используя отображение $\Theta$, легко показать, что в $B$ выполняется неградуированное тождество $\left(z_{1} z_{2}\right)\left(z_{3} z_{4}\right) \equiv 0$, т. е. $B$ - метабелева алгебра Ли. С другой стороны, очевидно, что $A$ и $G(B)=G(G(A))$ порождают одно и то же многообразие супералгебр Ли. Поскольку $c_{n}(B) \leqslant n$, то из предложения 2 получаем оценку $\gamma_{n}\left(\mathbf{A}^{2}\right) \leqslant 2^{n} n$. Таким образом, учитывая обе найденные оценки, получаем, что экспонента метабелева многообразия супералгебр Ли равна двум.

Напомним определение супералгебры Ли, которая порождает еще одно многообразие почти полиномиального роста. Алгебра $H=H_{0} \oplus H_{1}$ является четырехмерной супералгеброй Ли с базисом $a, b, u, v$ и таблицей умножения $a b=0$, $u v=0, a u=v, a v=u, b u=u, b v=v, a^{2}=2 b, b^{2}=u^{2}=v^{2}=0$, в которой $H_{0}=\langle b, v\rangle, H_{1}=\langle a, u\rangle$. Для многообразия супералгебр Ли, порожденного алгеброй $H$, в [1, лемма 3] доказан следующий результат.

ЛЕмма 4. Для любой константы $\alpha<\frac{2}{3}$ выполняются неравенства

$$
2^{\alpha n} \leqslant \gamma_{n}(\operatorname{var} H) \leqslant 2^{n} n^{2} .
$$

Уточним эту оценку и найдем экспоненту этого многообразия.

ТеОРема 5. Пусть $\mathbf{V}=\operatorname{var}(H)$ - многообразие, порожденное супералгеброй Ли Н. Тогда

$$
\frac{2^{n}}{n^{2}} \leqslant \gamma_{n}(\mathbf{V}) \leqslant 2^{n} n^{2}
$$

В частности, экспонента многообразия $\mathbf{V}$ равна двум, т.е. $\operatorname{Exp}(\mathbf{V})=2$. 
ДокАЗАтельство. Учитывая лемму 4, достаточно уточнить оценку снизу. Рассмотрим в свободной неассоциативной алгебре элемент $t_{1}=t_{1}\left(x_{0}, x_{1}, x_{2}\right)$ вида $t_{1}=\tilde{x}_{1}\left(\bar{x}_{1}\left(\left(x_{0} \bar{x}_{2}\right) \tilde{x}_{2}\right)\right)$. Напомним, что одинаковый символ (волна или черта) над парой переменных означает, что по ним проведено альтернирование. Заметим сначала, что

$$
\begin{aligned}
t_{1}(u, b, a) & =\tilde{b}\left(\bar{b}\left(\left(x_{0} \bar{a}\right) \tilde{a}\right)\right) \\
& =b(b((u a) a))+a(a((u b) b))-a(b((u a) b))-b(a((u b) a)) \\
& =b(b(v a))+a(a(-u b))-a(b(v b))-b(a(-u a)) \\
& =-b(b u)+a(a u)+a(b v)+b(a v)=-u+u+u+u=2 u .
\end{aligned}
$$

Теперь для произвольного $k \geqslant 2$ по индукции определим

$$
t_{k}\left(x_{0}, x_{1}, x_{2}\right)=\tilde{\tilde{x}}_{1}\left(\overline{\bar{x}}_{1}\left(\left(t_{k} \overline{\bar{x}}_{2}\right) \tilde{\tilde{x}}_{2}\right)\right) .
$$

Тогда $t_{k}(u, b, a)=2^{k} u \neq 0$, т. е. $t_{k}$ не является тождеством многообразия $\mathbf{V}$ ни при каком $k \geqslant 1$. Так как $\operatorname{deg} t_{k}=4 k+1$ и $t_{k}$ содержит $2 k$ кососимметричных пар одинаковых переменных, то для любого $n=4 k+1$ линеаризация элемента $t_{k}\left(x_{0}, x_{1}, x_{2}\right)$ порождает в полилинейной части $P_{n}(\mathbf{V})$ модуль, содержащий ненулевой неприводимый подмодуль, соответствующий разбиению $\lambda=(2 k+1,2 k)$ или $\lambda=(2 k, 2 k, 1)$. В любом случае из формулы крюков получаем

$$
\gamma_{n}(\mathbf{V}) \geqslant \frac{2^{n}}{n^{2}} .
$$

Теорема доказана.

В заключение напомним, что для градуированных алгебр возможен и другой подход к определению функции роста. Если $L=L_{0} \oplus L_{1}=\operatorname{alg}\left\{x_{1}, x_{2}, \ldots\right.$ $\left.\ldots, y_{1}, y_{2}, \ldots\right\}$ - относительно свободная супералгебра счетного ранга некоторого многообразия $\mathbf{V}, x_{i} \in L_{0}, y_{i} \in L_{1}$, то можно рассмотреть пространство $P_{m, k}(\mathbf{V})$ полилинейных полиномов от $x_{1}, \ldots, x_{m}, y_{1}, \ldots, y_{k}$. Обозначим

$$
P_{n}^{\prime}(\mathbf{V})=\sum_{k=0}^{n} P_{k, n-k}(\mathbf{V})
$$

и положим

$$
\mu_{n}(\mathbf{V})=\operatorname{dim} P_{n}^{\prime}(\mathbf{V})=\sum_{k=0}^{n} \operatorname{dim} P_{k, n-k}(\mathbf{V})
$$

Тогда последовательность $\left\{\mu_{n}(\mathbf{V})\right\}, n=1,2, \ldots$, также характеризует многообразие $\mathbf{V}$ и ее можно взять в качестве другой функции роста, отличной от $\gamma_{n}(\mathbf{V})$. Именно эта функция роста рассматривалась в работе [16]. Для метабелева многообразия в этой работе показано, что $\mu_{n}\left(\mathbf{A}^{2}\right)=n^{2}-1$, а $\gamma_{n}\left(\mathbf{A}^{2}\right)$ растет экспоненциально.

\section{Список литературы}

1. М.В. Зайцев, С. П. Мищенко, "Критерий полиномиальности роста многообразий супералгебр Ли”, Изв. РАН. Сер. матем., 62:5 (1998), 103-116; англ. пер.: M. V. Zaitsev, S. P. Mishchenko, "A criterion for polynomial growth of varieties of Lie superalgebras", Izv. Math., 62:5 (1998), 953-967. 
2. A. Regev, "Existence of identities in $A \otimes B$ ", Israel J. Math., 11 (1972), 131-152.

3. И.Б. Воличенко, "О многообразии алгебр Ли $A N_{2}$ над полем характеристики нуль", Докл. АН БССР, 25:12 (1981), 1063-1066.

4. В. М. Петроградский, "О типах сверхэкспоненциального роста тождеств в PIалгебрах Ли", Фундам. и прикл. математика, 1:4 (1995), 989-1007.

5. V. Drensky, A. Regev, "Exact asympotic behaviour of the codimensions of some PIalgebras", Israel J. Math., 96 (1996), 231-242.

6. С. П. Мищенко, "О многообразиях алгебр Ли промежуточного роста", Вести АH БССР, 1987, № 2, 42-45.

7. А.Р. Кемер, "Шпехтовость $T$-идеалов с полиномиальным ростом коразмерностей", Сиб. матем. журн., 19:1 (1978), 54-69; англ. пер.: А. R. Kemer, "Spechtianess of $T$-ideals with polynomial growth of the co-dimensions", Siberian Math. J., 19:1 (1978), 37-48.

8. И. И. Бенедиктович, А. Е. Залесский, “Т-идеалы свободной алгебры Ли с полиномиальным ростом последовательности коразмерностей”, Вести АН БССР, 1981, № $3,5-10$.

9. С.П. Мищенко, "О многообразиях полиномиального роста алгебр Ли над полем характеристики нуль", Матем. заметки, 40:6 (1986), 713-721; англ. пер.: S.P. Mishchenko, "Varieties of polynomial growth of Lie algebras over a field of characteristic zero", Math. Notes, 40:6 (1986), 901-905.

10. В.С. Дренски, "Представления симметрической группы и многообразия линейных алгебр", Матем. сб., 115:1 (1981), 98-115; англ. пер.: V.S. Drenski, "Representations of the symmetric group and varieties of linear algebras", Math. USSR-Sb., 43:1 (1982), 85-101.

11. Д.В. Репин, "Градуированные тождества простой трехмерной алгебры Ли", Вестн. СамГУ, 2-й спец. вып., 2004, 5-16.

12. Ю. П. Размыслов, “О конечной базируемости тождеств матричной алгебры второго порядка над полем характеристики нуль”, Алгебра и логика, 12:1 (1973), 83-113; англ. пер.: Yu. P. Razmyslov, "Finite basing of the identities of a matrix algebra of second order over a field of characteristic zero", Algebra and Logic, 12:1 (1973), 47-63.

13. Ю. П. Размыслов, "Конечная базируемость некоторых многообразий алгебр", $А л$ гебра и логика, 13:6 (1974), 685-693; англ. пер.: Yu. P. Razmyslov, "Finite basis property for certain varieties of algebras", Algebra and Logic, 13:6 (1974), 394-399.

14. J.-P. Serre, Représentations linéaires des groupes finis, Hermann, Paris, 1967.

15. М. В. Зайцев, С. П. Мищенко, "О кодлине многообразий линейных алгебр", $M a-$ тем. заметки, 79:4 (2006), 553-559; англ. пер.: M. V. Zaitsev, S. P. Mishchenko, "Colength of varieties of linear algebras", Math. Notes, 79:3-4 (2006), 511-517.

16. Ю.А. Бахтурин, В.С. Дренски, "Тождества разрешимых цветных супералгебр Ли", Алгебра и логика, 26:4 (1987), 403-418; англ. пер.: Yu. A. Bakhturin, V.S. Drenski, "Identities of solvable color Lie superalgebras", Algebra and Logic, 26:4 (1987), 229-240.

M. В. ЗАЙЦЕв (M. V. ZAitsev)

Поступило в редакцию

Московский государственный университет

14.11.2005

им. М. В. Ломоносова

E-mail: zaicev@mech.math.msu.su

С. П. Мищенко (S. P. Mishchenko)

Ульяновский государственный университет

E-mail: mishchenkosp@ulsu.ru 